\title{
Therapeutic application of extracellular vesicles for various kidney diseases: a brief review
}

\author{
Sul A Lee ${ }^{1} \&$ Tae Hyun Yoo ${ }^{2, *}$ \\ ${ }^{1}$ Department of Medicine, MetroWest Medical Center/Tufts University School of Medicine, Framingham, MA 01702, USA, ${ }^{2}$ Department of \\ Internal Medicine, College of Medicine, Institute of Kidney Disease Research, Yonsei University, Seoul 03722, Korea
}

Extracellular vesicles (EVs) released from different types of kidney cells under physiologic conditions contribute to homeostasis maintenance, immune-modulation, and cell-to-cell communications. EVs can also negatively affect the progression of renal diseases through their pro-inflammatory, pro-fibrotic, and tumorigenic potential. Inhibiting EVs by blocking their production, release, and uptake has been suggested as a potential therapeutic mechanism based on the significant implication of exosomes in various renal diseases. On the other hand, stem cell-derived EVs can ameliorate tissue injury and mediate tissue repair by ameliorating apoptosis, inflammation, and fibrosis while promoting angiogenesis and tubular cell proliferation. Recent advancement in biomedical engineering technique has made it feasible to modulate the composition of exosomes with diverse biologic functions, making EV one of the most popular drug delivery tools. The objective of this review was to provide updates of recent clinical and experimental findings on the therapeutic potential of EVs in renal diseases and discuss the clinical applicability of EVs in various renal diseases. [BMB Reports 2022; 55(1): 3-10]

\section{INTRODUCTION}

Extracellular vesicles (EVs) are endogenously produced, membrane-bound vesicles that are released from cells into the extracellular space (1). Exosomes and microvesicles (MVs) are major subtypes of EVs. They are known to serve important roles in homeostasis, immune modulation, and tissue regeneration under physiologic conditions $(2,3)$. EVs can also mediate inflammation, thrombosis, fibrosis, and tumorigenesis in pathologic conditions $(4,5)$. EVs contain various biologic materials including

*Corresponding author. Tel: +82-2-2228-1975; Fax: +82-2-393-6884; E-mail: yoosy0316@yuhs.ac

https://doi.org/10.5483/BMBRep.2022.55.1.141

Received 13 September 2021, Revised 17 November 2021, Accepted 10 December 2021

Keywords: Drug delivery, Exosome, Extracellular vesicles, Kidney, Microvesicles, Renal disease
mRNA, microRNA (miRNA), proteins, and lipids. Their contents are determined by the type of host cells and microenvironments of host cells (6). The biologic function of EVs depends on their compositions and downstream responses of recipient cells.

EVs serve several pivotal roles in renal physiology including immune modulation, tissue proliferation/regeneration, antimicrobial effect, and electrolyte/water balance, which contributes to maintenance of renal homeostasis (3). In pathologic conditions, however, EVs can contribute to propagation of disease courses by enhancing inflammation, fibrosis, coagulation, and tumorigenesis in various renal disease conditions (3). The role of EVs as a novel biomarker gained a lot of attention and comprised a major proportion of EV studies in kidneys. This topic is well summarized in the review article by Karpman et al. (1) and this will not be addressed further in this review as it is beyond the scope of our topic.

Based on the recent advancement of stem cell research and biomedical engineering technique on EV loading and modification (7), EVs have received a lot of medical attention for treatment of various kidney diseases including AKI, CKD, and transplant graft rejection even though further verification through human studies is limited. EVs serve a crucial role in intercellular communication by delivering biological cargo to recipient cells. The potential use of EVs as biocarriers has been exploited for the delivery of endogenous or exogenous therapeutic materials (3). Diverse cargos including miRNAs, proteins, and drugs, can be delivered to target cells by modulating EV production and cargo sorting. In this review, we will focus on the potential of EVs as intrinsic therapeutics, therapeutic targets, and drug carriers for various renal diseases.

\section{EVs FOR TREATING VARIOUS KIDNEY DISEASES}

More recently, both in vivo and in vitro studies have shown explosive advancements regarding the protective role of EVs in various types of renal diseases. Most EVs used in those studies originated from mesenchymal stem cells (MSCs). They can alleviate renal damage mainly through their paracrine effects rather than their differentiation potential (8). MSC-derived EVs can exert therapeutic effects by modulating various biological processes including tubular proliferation, angiogenesis, apoptosis, 
and fibrosis (Fig. 1). Below, we will review the therapeutic potential of EVs in renal disease conditions in more detail which is also summarized in Table 1.

\section{Acute kidney injury (AKI)}

$\mathrm{AKI}$ is a clinical syndrome originating from acute loss of renal excretory function and typically results in accumulation of renal toxins or reduction in urine output. AKI can originate from various etiologies including dehydration, toxins, hemodynamic instability, or obstruction. AKI is associated with increased mortality and healthcare-related costs. Therefore, many studies have focused on finding novel therapeutics using EVs to prevent or improve AKI outcome which will be addressed more in detail below.

MSC-derived EVs have shown a profound protective effect on AKI through their anti-apoptotic, antioxidant, anti-inflammatory, and angiogenic activities. In a study by Chen et al. (9), MVs derived from human Wharton's Jelly mesenchymal stromal cells (hWJMSCs) could ameliorate renal ischemia reperfusion injury (IRI) by enhancing the regeneration and decreasing the apoptosis of renal cells while mitigating IR-induced renal fibrosis. This protection was induced by G2/M cell cycle arrest via Erk1/2 signaling. Another study has shown that hWJMSCderived MVs possess antioxidant effects in a rat ischemic AKI model (10). A single administration of hWJMSC-derived MVs into IR-injured kidneys decreased the expression of reactive oxygen species, reduced apoptosis, and enhanced proliferation of tubular cells in vivo. Renal fibrosis in the late stage of IRI was also significantly abrogated by MV delivery, leading to similar biochemical improvement (10).

As identification and characterization of noncoding RNAs become more widely available, several studies have shown therapeutic potentials of exosomal miRNAs in ischemic AKI by post-transcriptional regulation. Li et al. (11) have found that both human urine-derived stem cells (USC) and their exosomes could protect ischemic AKI in mice. Further sequencing and bioinformatics analysis showed that miR-146a-5p was the most abundant miRNA in USC-derived exosomes. They found that miR-146a-5p targeted interleukin (IL)-1 receptor-associated kinase 1 mRNA, subsequently inhibiting the activation of NF-KB signaling in vitro (11). Zhu et al. (12) have demonstrated that exosomes from human-bone-marrow-derived MSCs can induce anti-apoptotic effect in ischemic AKI by transferring miR-199a$3 p$ to renal cells. This protective effect was mediated via modulation of protein kinase B and extracellular-signal-regulated kinase pathways (12). However, neither of these studies traced or targeted exosomes in vivo. Thus, direct visualization of exosome transfer into target renal cells could not be provided by these studies.

Several studies have shown therapeutic effects of EVs derived from various sources other than MSCs $(13,14)$. Pan et al. (13) have found that the protective effect of limb remote ischemic preconditioning (rIPC) is mediated by miR-21 transportation from preischemic limbs to remote organs via serum exosomes. Serum-derived exosomes from mice with limb rIPC or enhanced exosomal miR-21 from cultured myotubes with hypoxia and reoxygenation preconditioning integrated into renal tubular epithelial cells and targeted downstream PDCD4/NF- $\mathrm{KB}$ and

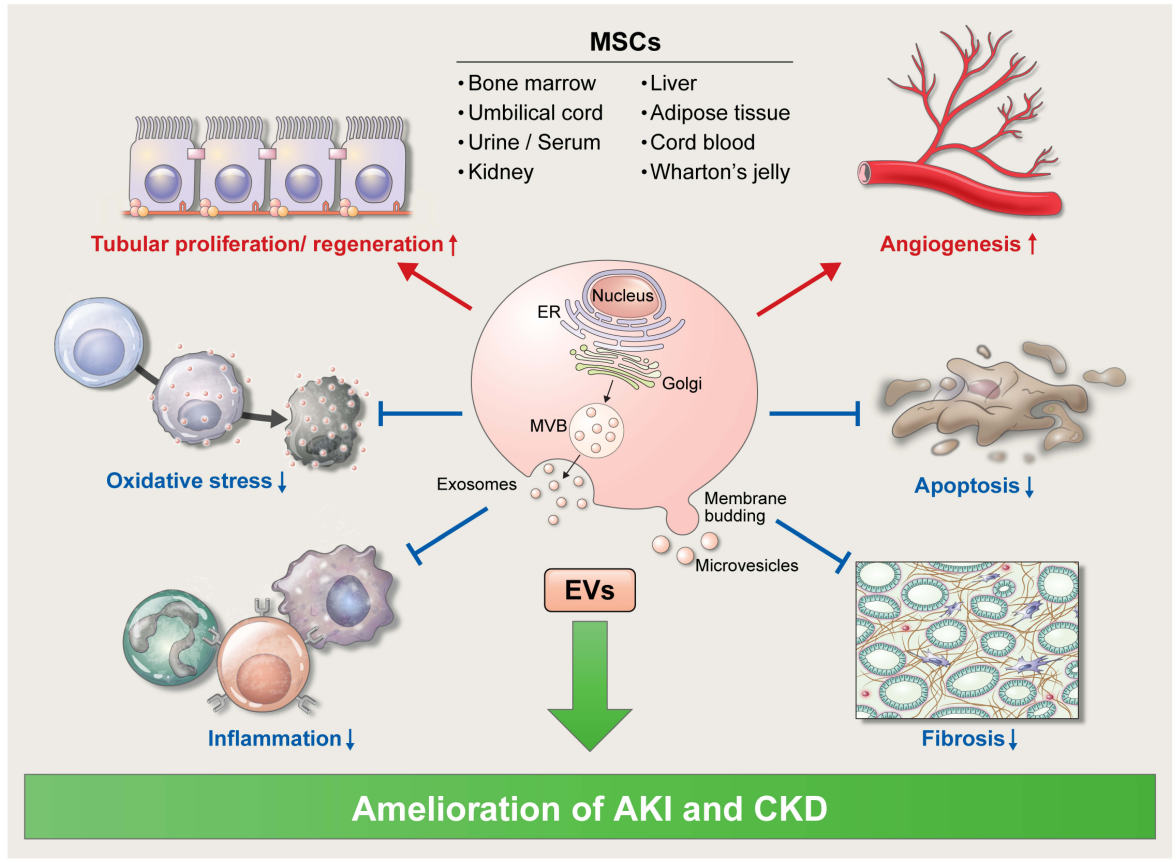

Fig. 1. MSC-derived EVs can ameliorate the course of AKI and CKD through modulation of various biological processes including promotion of tubular proliferation and angiogenesis, alleviation of oxidative stress, and reduction of inflammation, apoptosis, and fibrosis. MSC: mesenchymal stromal cells, EVs: extracellular vesicles, AKI: acute kidney injury, CKD: chronic kidney disease, ER: endoplasmic reticulum, MVB: multivesicular body. 
Table 1. Therapeutic application of extracellular vesicles from various origins in different kidney diseases

\begin{tabular}{|c|c|c|c|c|}
\hline Disease Model & Origin & EV type & Mechanism & Ref. \\
\hline \multirow[t]{11}{*}{ AKI } & hWJMSCs & $\mathrm{MVs}$ & $\begin{array}{l}\text { - hWJMSC-derived MVs improve renal function in ischemic AKI model by } \\
\text { facilitating the proliferation of renal tubular cells and alleviating the apoptosis and } \\
\text { fibrosis of renal cells in vivo (rats). }\end{array}$ & (9) \\
\hline & hWJMSCs & Not specified & $\begin{array}{l}\text { - hWJMSC-derived EVs ameliorate ischemic AKI by inhibition of mitochondrial fission } \\
\text { through miR-30 in vivo (rats). }\end{array}$ & (44) \\
\hline & hWJMSCs & MVs & $\begin{array}{l}\text { - hWJMSC-derived MVs alleviate the oxidative stress through } \\
\text { suppressing NOX2 expression in both in vitro (HUVEC) and in vivo (rats) IRI model. } \\
\text { - hWJMSC-derived MVs reduce apoptosis and enhanced proliferation in renal IRI. }\end{array}$ & (10) \\
\hline & hWJMSCs & MVs & $\begin{array}{l}\text { - hWJMSC-derived MVs induce HGF synthesis in damaged tubular cells via } \\
\text { RNA transfer, facilitating tubular cell dedifferentiation and regeneration in unilateral } \\
\text { AKI model in vivo (rats). }\end{array}$ & (45) \\
\hline & hUC-MSCs & MVs & $\begin{array}{l}\text { - hUC-MSC-derived MVs mitigate epithelial cell apoptosis in low oxygen environment } \\
\text { in vitro (HK-2) and ameliorated renal IRI in vivo (rats) via delivery of miR-21. }\end{array}$ & (46) \\
\hline & hUSCs & Exosomes & $\begin{array}{l}\text { - hUSC-derived exosomes ameliorate ischemic AKI in vivo (rats). } \\
\text { - hUSC-derived exosomes inhibit oxidative stress after H/R injury in vitro (HK-2). } \\
\text { - miR-146a-5p targets interleukin-1 receptor-associated kinase } 1 \text { mRNA and } \\
\text { subsequently inhibited the activation of NF-KB signaling in vitro (HK-2). }\end{array}$ & (11) \\
\hline & $\begin{array}{l}\text { hBM- } \\
\text { derived } \\
\text { MSCs }\end{array}$ & Exosomes & $\begin{array}{l}\text { - Exosomes from hBM-derived MSCs play a protective role in H/R injury in vitro (HK-2) } \\
\text { as well as in renal IRI in vivo (mice). } \\
\text { - miR-199a-3p is involved in the renal protective effects of exosomes from } \\
\text { hBM-derived MSCs by regulating Sema3A and activating the AKT and ERK pathways. }\end{array}$ & (12) \\
\hline & $\begin{array}{l}\text { Adipose- } \\
\text { derived } \\
\text { MSCs }\end{array}$ & Not specified & $\begin{array}{l}\text { - Hypoxia-preconditioning increase the antiapoptotic, immune-modulatory, } \\
\text { and anti-oxidative properties of adipose-derived MSC-EVs in vitro. } \\
\text { - Adipose-derived MSC-EVs improve recovery of renal function in ischemic AKI in vivo (rats). }\end{array}$ & (47) \\
\hline & Mouse serum & Exosomes & $\begin{array}{l}\text { - Delayed remote ischemic preconditioning exerts renoprotection in septic AKI through } \\
\text { exosomal miR-21 derived from preischemic limbs in vivo (mice). } \\
\text { - Exosomal miR-21 attenuates septic AKI both in vivo (mice) and in vitro (mTECs) } \\
\text { through PDCD4/NF-KB and PTEN/AKT pathways inducing anti-inflammatory and } \\
\text { anti-apoptotic effects. }\end{array}$ & (13) \\
\hline & Human urine & Not specified & $\begin{array}{l}\text { - Urinary EVs alleviate AKI generated by glycerol injection and accelerate renal recovery } \\
\text { in vivo (mice). } \\
\text { - The protective role of urinary EV is mediated through repletion of Klotho in } \\
\text { injured renal tissue. }\end{array}$ & (14) \\
\hline & $\begin{array}{l}\text { Human renal } \\
\text { tubular cells }\end{array}$ & Exosomes & $\begin{array}{l}\text { - Exosomes from human renal tubular cells prevent ischemic renal injury in Nude rats } \\
\text { by preventing renal oxidant stress and apoptosis and suppressing pro-inflammatory } \\
\text { and pro-fibrotic pathways. }\end{array}$ & (16) \\
\hline \multirow[t]{4}{*}{$\begin{array}{l}\text { Diabetic } \\
\text { nephropathy }\end{array}$} & $\begin{array}{l}\text { Rat } \\
\text { BM-derived } \\
\text { MSCs }\end{array}$ & Exosomes & $\begin{array}{l}\text { - BM-derived exosomes improve renal function, morphology, and fibrosis in } \\
\text { streptozotocin-induced diabetic nephropathy model in vivo (rats) in parallel with } \\
\text { increased autophagy markers, LC3 and Beclin-1, and decreased mTOR and } \\
\text { fibrotic markers expression in renal tissue. }\end{array}$ & (18) \\
\hline & $\begin{array}{l}\text { Rat } \\
\text { BM-derived } \\
\text { MSCs }\end{array}$ & Exosomes & $\begin{array}{l}\text { - Exosomes from BM-derived MSCs ameliorate renal inflammation and fibrosis while } \\
\text { protecting tight junction structure in streptozotocin-induced diabetic nephropathy } \\
\text { in vivo (rats). } \\
\text { - Exosomes from BM-derived MSCs suppress apoptosis and degeneration of } \\
\text { tubular epithelial cells in primary renal cell culture of streptozotocin-induced diabetic } \\
\text { rats in vitro. }\end{array}$ & (19) \\
\hline & hUC-MSCs & Exosomes & $\begin{array}{l}\text { - hUC-MSC-derived exosomes decrease the production of pro-inflammatory and } \\
\text { pro-fibrotic cytokines in high glucose-injured renal tubular epithelial cells and } \\
\text { renal glomerular endothelial cells in vitro. }\end{array}$ & (48) \\
\hline & $\begin{array}{l}\text { hBM-derived } \\
\text { MSCs and } \\
\text { HLSCs }\end{array}$ & Not specified & $\begin{array}{l}\text { - EVs from hBM-derived MSCs and HLSCs alleviate renal fibrosis and proteinuria in } \\
\text { streptozotocin-induced diabetic nephropathy model in vivo (mice). }\end{array}$ & (49) \\
\hline \multirow[t]{2}{*}{$\begin{array}{l}\text { Hypertensive } \\
\text { nephropathy }\end{array}$} & $\begin{array}{l}\text { Adipose- } \\
\text { derived } \\
\text { MSCs }\end{array}$ & Not specified & $\begin{array}{l}\text { - Adipose-derived MSC-EVs improve renal function, decreased urinary protein } \\
\text { excretion, and renal fibrosis while preventing cardiac tissue fibrosis and inducing } \\
\text { better blood pressure control in DOCA-salt hypertensive model in vivo (rats). }\end{array}$ & (20) \\
\hline & $\begin{array}{l}\text { Cardiosphere- } \\
\text { derived } \\
\text { cells }\end{array}$ & Exosomes & $\begin{array}{l}\text { - Administration of exosomes from cardiosphere-derived cells attenuate renal injury and } \\
\text { cardiac hypertrophy in angiotensin II-induced hypertension model in vivo (mice), } \\
\text { which appears to be associated with changes in the expression of interleukin-10. }\end{array}$ & (21) \\
\hline
\end{tabular}


Table 1. Continued

\begin{tabular}{|c|c|c|c|c|}
\hline Disease Model & Origin & EV type & Mechanism & Ref. \\
\hline $\begin{array}{l}\text { Glomerulone- } \\
\text { phritis }\end{array}$ & hEPC & Not specified & $\begin{array}{l}\text { - hEPC-derived EVs alleviate complement-mediated mesangial injury in } \\
\text { anti-Thy1.1-induced glomerulonephritis model in vivo (rats) by } \\
\text { inhibiting mesangial cell activation, leukocyte infiltration, and apoptosis. } \\
\text { - hEPC-derived EVs inhibit complement-mediated renal mesangial cell injury and } \\
\text { C5b-9 deposition in vitro. }\end{array}$ & (22) \\
\hline \multirow[t]{6}{*}{ Other CKD } & $\begin{array}{l}\text { Adipose- } \\
\text { derived } \\
\text { autologous } \\
\text { MSCs }\end{array}$ & Not specified & $\begin{array}{l}\text { - Autologous MSCs-derived EVs restore renal function through attenuation of } \\
\text { renal inflammation, tissue hypoxia, and fibrosis in metabolic syndrome and } \\
\text { renal artery stenosis model in vivo (pigs). } \\
\text { - These protective effects are blunted in pigs treated with interleukin-10-depleted EVs. }\end{array}$ & (23) \\
\hline & MSCs & Not specified & $\begin{array}{l}\text { - MSC-derived EVs from lean pigs more effectively improve renal function and } \\
\text { decrease tubular injury and fibrosis compared to those from pigs with } \\
\text { metabolic syndrome. } \\
\text { - The beneficial effect of MSC-derived EVs appears to be associated with } \\
\text { up-regulated TGF- } \beta \text { signaling and enriched regulatory T cells. }\end{array}$ & (24) \\
\hline & hCB-MSCs & Not specified & $\begin{array}{l}\text { - Cell-free hCB-MSCs-EVs ameliorate the inflammatory immune reaction and } \\
\text { transiently improve the overall kidney function in CKD patients. } \\
\text { - Cell-free hCB-MSCs-EVs do not induce any significant adverse events throughout } \\
\text { the study period (one year). }\end{array}$ & (50) \\
\hline & $\begin{array}{l}\text { hBM- } \\
\text { derived } \\
\text { MSCs }\end{array}$ & Exosomes & $\begin{array}{l}\text { - MSCs-derived exosomal anti-let-7i-5p attenuates the pro-fibrotic response induced by } \\
\text { TGF- } \beta 1 \text { in vitro (NRK52E cells). } \\
\text { - MSC-derived exosomal anti-let-7i-5p improves renal function and } \\
\text { attenuates renal fibrosis in UUO-induced renal fibrosis model in vivo (mice). }\end{array}$ & (51) \\
\hline & hWJMSCs & MV & $\begin{array}{l}\text { - hWJMSC-derived MVs attenuate ischemia-induced renal fibrosis in vivo (rats) and } \\
\text { promote M2 macrophage polarization in vitro (THP-1 macrophages) via } \\
\text { transferring HGF. }\end{array}$ & (52) \\
\hline & $\begin{array}{l}\text { Human } \\
\text { adipose- } \\
\text { derived } \\
\text { MSCs }\end{array}$ & Exosomes & $\begin{array}{l}\text { - GDNF-modified human adipose-derived MSCs ameliorate renal fibrosis in } \\
\text { murine UUO model. } \\
\text { - GDNF-modified human adipose-derived MSCs exert cytoprotective effect on HUVEC } \\
\text { in hypoxia/serum deprivation injury model by promoting angiogenesis through } \\
\text { activation of SIRT1/eNOS signaling pathway. }\end{array}$ & (53) \\
\hline \multirow{3}{*}{$\begin{array}{l}\text { Graft } \\
\text { dysfunction } \\
\text { after renal } \\
\text { trans- } \\
\text { plantation }\end{array}$} & Tregs & Exosomes & $\begin{array}{l}\text { - Treg-derived exosomes can postpone allograft rejection and prolong the survival time } \\
\text { of transplanted kidney in vivo (rats). } \\
\text { - Treg-derived exosomes suppress T cell proliferation in vitro. }\end{array}$ & (26) \\
\hline & $\begin{array}{l}\text { Mouse } \\
\text { immature } \\
\text { DCs }\end{array}$ & Exosomes & $\begin{array}{l}\text { - Immature DC-derived exosomes improve the survival in isograft mice by } \\
\text { alleviating inflammatory response, reducing CD4 T cell infiltration, } \\
\text { and increasing regulatory T cells in spleen and kidney tissues. } \\
\text { - miR-682 is highly expressed in immature DC-derived exosomes which } \\
\text { can promote regulatory T cell differentiation and immune tolerance in } \\
\text { renal allograft in vivo (mice). }\end{array}$ & (27) \\
\hline & hWJMSCs & MV & $\begin{array}{l}\text { - hWJMSC-derived MVs improve survival rate and renal function after } \\
\text { renal transplantation in vivo (rats). } \\
\text { - hWJMSC-derived MVs mitigate renal cell apoptosis and inflammation and } \\
\text { enhance proliferation in the acute stage while abrogating renal fibrosis in the late stage. }\end{array}$ & (28) \\
\hline
\end{tabular}

EV: extracellular vesicles, AKI: acute kidney injury, hWJMSCs: human Wharton's Jelly mesenchymal stromal cells, MVs: microvesicles, miR: microRNA, HUVEC: human umbilical vein endothelial cells, IRI: ischemia-reperfusion injury, HGF: hepatocyte growth factor, hUC-MSCs: human umbilical cord mesenchymal stem cells, HK-2: human tubule epithelial cells, hUSCs: human urine-derived stem cells, H/R: hypoxia/reoxygenation, hBM: human bone marrow, MSCs: mesenchymal stem cells, AKT: protein kinase B, ERK: extracellular signal-regulated kinase, mTECs: mouse tubular epithelial cells, mTOR: mammalian target of rapamycin, HLSCs: human liver stem-like cells, CKD: chronic kidney disease, DOCA: deoxycorticosterone acetate, TGF- 31 : transforming growth factor beta-1, hCB-MSCs: human cord blood mesenchymal stem cells, UUO: unilateral ureteral obstruction, GDNF: Glial cell line-derived neutrophic factor, SIRT1: Sirtuin 1, eNOS: endothelial nitric oxide synthase, hEPC: human endothelial progenitor cells, Tregs: regulatory T cells, DCs: dendritic cells.

PTEN/AKT pathways. The delivery of those exosomes attenuated sepsis-induced AKI through their anti-inflammatory and anti-apoptotic effects. In a glycerol-induced AKI model, the delivery of urine-derived EVs (uEVs) from healthy volunteers alleviated biochemical and histological renal injury, improved tubular cell proliferation, and reduced tubular cell apoptosis (14). Biodistribution analysis confirmed the preferential localization of uEVs in damaged kidneys. Treatment with human $\mathrm{uEV}$ s could restore Klotho to normal levels in injured kidneys which is known to serve reno-protective role in AKI by inhibiting apoptosis, fibrosis 
and upregulating autophagy (15). However, uEVs from Klotho null mice did not show any reno-protective effect. On the other hand, Klotho engineered uEVs from Klotho null mice restored regenerative properties, suggesting the indispensable role of Klotho in the protective mechanism of uEVs. Dominguez et al. (16) have shown that exosomes from human renal tubules could reverse renal IRI in nude rats through maintenance of renal vascular and epithelial networks, protection from oxidative stress and apoptosis, and suppression of pro-inflammatory and pro-fibrotic pathways. Further comprehensive proteomic analysis on IR-injured kidneys showed that renal IRI induced significant and extensive changes in protein expression. However, treatment with human renal tubular exosomes could prevent most of these protein expression alterations (16).

\section{Chronic kidney disease (CKD)}

Renal fibrosis is a major contributor to CKD pathophysiology and can cause irreversible deterioration of renal function. The severity of renal fibrosis is significantly correlated with progression of CKD. Pathways, diagnostic potential, and therapeutic potential of EV-regulated renal fibrosis in CKD are well described in a review article by Brigstock (17). Here, we will review some representative in vivo studies regarding therapeutic actions of $\mathrm{EV} \mathrm{s}$ in various experimental models of CKD.

The most common etiology of CKD is diabetic nephropathy, a microvascular complication from hyperglycemia-induced oxidative injury and inflammation that can ultimately lead to renal fibrosis. MSC-derived exosomes have shown renal-protective effects on diabetic nephropathy $(18,19)$, although the exact mechanism has not been completely understood. Using a rat model of streptozotocin-induced diabetes mellitus model, Ebrahim et al. (18) have reproduced improved biochemical and histological renal outcomes in a group treated with MSC-treated exosomes compared to a control group. Treatment with MSCderived exosomes induced significant upregulation of autophagy markers, Beclin-1, and light chain-3, and downregulated mechanical target of rapamycin (mTOR) and fibrotic marker expression in renal tissues (18). The protective effect of MSC-derived exosomes was partially reversed by administration of autophagy inhibitors, suggesting that autophagy induction by exosomes could attenuate diabetic nephropathy (18). MSC-derived exosomes could also exert anti-apoptotic, anti-fibrotic, and anti-degenerative effects in tubular epithelial cells while protecting tight junction structure in streptozotocin-induced diabetic nephropathy model of rats (19).

Hypertension is the second leading etiology of CKD, causing damage to blood vessels and filtering function of the kidney. In a deoxycorticosterone acetate-salt hypertensive model, EVs from adipose-derived MSCs could ameliorate pro-inflammatory response and recruitment of immune cells into the kidney (20). Moreover, administration of these EVs could prevent cardiac tissue fibrosis and induce better blood pressure control. Further miRNA microarray profile suggested that EV administration could affect signaling pathway of epithelial-mesenchymal transition and pre- vent inflammation as well as fibrosis in the kidney. In an angiotensin Il-induced hypertensive model, exosomes from cardiosphere-derived cells improved renal function and cardiac hypertrophy while diminishing inflammation and fibrosis in both kidney and heart in association with altered levels of IL-10 expression (21).

A study by Cantaluppi et al. showed that EVs from endothelial progenitor cells can decrease antibody- and complementmediated injury in Thy1.1-treated glomerulonephritis model (22). This protective effect was significantly reduced by pretreatment with a high dose RNase, suggesting a crucial role of RNA content in EVs. In a pig model of metabolic syndrome and renal artery stenosis, exosomes from autologous MSCs could preserve renal function by alleviating renal inflammation, tissue hypoxia, and fibrosis (23). This reno-protective capacity appeared to be mediated by exosomal IL-10, an anti-inflammatory cytokine. Song et al. (24) have shown that MSC-derived EVs from lean pigs are more effective in improving renal function and decreasing tubular injury and fibrosis than EVs from metabolic syndrome pigs. These beneficial effects were associated with enhanced anti-inflammatory transforming growth factor (TGF)- $\beta$ signaling leading to regulatory $\mathrm{T}$ cell induction (24).

\section{Graft dysfunction after renal transplantation}

Renal transplant has become the treatment of choice for most of the advanced kidney disease by placing a healthy kidney from a donor into a recipient's body. Transplant procedure itself induces some degree of ischemic-reperfusion damage as well as tissue damage which has significant impact on early graft function (25). Long-term immunosuppressive treatment is also crucial to prevent graft rejection and to prolong the graft survival as well as its function maintenance. EVs are known to serve a various role in transplanted kidney through their modulatory functions in innate immunity, complement system, and coagulation system, either by activating or inhibiting them depending on the microenvironment and EV content (25). EVs are also involved in allorecognition, IRI, and the autoimmune component of antibody-mediated rejections, affecting on the graft function and survival (25). Kidney endothelial- and tubular-derived $\mathrm{EV}$ s can trigger graft rejection by inducing alloimmune and autoimmune responses, while MSC-derived EVs have been investigated for their therapeutic potential in experimental transplant models (26-28). The role of EVs in the crosstalk between the renal graft and immune systems as well as the diagnostic and therapeutic role of EVs in renal transplantation are well summarized in the review article by Quaglia et al. (25).

In a rat model of kidney transplantation, exosomes derived from regulatory $T$ cells could delay allograft rejection, prolong the survival time of transplanted kidney, and inhibit $\mathrm{T}$ cell proliferation (26). This protective effect was more prominent by using the exosomes collected from donors compared to those from recipients. Pang et al. (27) have shown that immature dendritic cells-derived exosomes could significantly improve 
graft survival by alleviating inflammatory response and regulating $T$ cell differentiation in renal grafts. They also found that immature dendritic cells-derived exosomes highly expressed miR-682 and that their protective effects are mediated through miR-682 to regulate $T$ cell differentiation by negatively affecting Rho-associated kinase 2 expression. In a rat model of renal transplantation from cardiac death donors, when hWJMSCs-derived $\mathrm{MVs}$ were injected immediately after renal transplantation, they improved graft survival and renal function both in acute and chronic stages by mitigating renal cell apoptosis, inflammation, and renal fibrosis (28).

\section{EVs AS POTENTIAL THERAPEUTIC TARGETS}

EVs exhibit potent effects in processes of thrombosis, inflammation, and apoptosis and are involved in propagation of various renal diseases. Therefore, blocking the release and uptake of exosomes can potentially carry beneficial effects during the disease course, even if the blocking is temporary. Various pharmacological agents can block release and uptake of EVs, including antiplatelet agents, statins, calcium channel blockers, and abciximab (29-31). However, whether modifying the release and uptake of exosomes can affect outcomes of renal diseases has not been fully investigated yet. Mossberg et al. (32) have shown that patients with acute vasculitis have markedly higher levels of kinin B1-receptor-positive endothelial MVs known to carry significant neutrophil chemotactic effects. Coincubation of plasma with C1-inhibitor, the main inhibitor of the kinin system, could significantly lower the release of kinin B1-receptor-positive endothelial MVs in vitro, indicating a therapeutic potential of $\mathrm{C} 1$-inhibitor in the treatment of inflammatory diseases such as vasculitis (32). Liu et al. (33) have shown that inhibition of exosome release could prevent kidney fibrosis in murine unilateral IRI and unilateral ureteral obstruction (UUO) models in vivo. Through pharmacologic inhibition of exosome secretion from renal proximal tubular cells using dimethyl amiloride (DMA), TGF $\beta 1$-treated renal tubular cells lost their ability to activate renal interstitial fibroblast in vitro (33). This study also showed that blocking exosome secretion using DMA or knockdown of Rab27a, an essential protein for exosome formation, could preserve kidney function and attenuate renal fibrosis in unilateral IRI and UUO models. Of note, neither DMA treatment nor Rab27a knockout caused any noticeable abnormality in normal kidney or affected the integrity of contralateral kidneys after unilateral IRI and UUO. This study suggests a novel avenue for developing therapeutic strategies against CKD by targeting biogenesis and secretion of exosomes.

\section{EVs AS BIOCARRIERS OF THERAPEUTIC MATERIALS}

Better understanding of biological mechanisms of EVs and simultaneous advancement of bio-engineering technology to modulate EV production and cargo sorting have made EV one of the most preferred drug delivery systems. There are multiple biological benefits of EVs as vectors over other methods, including their stability, reduced toxicity, biostability, and low immunogenicity (34). Specific cell surface molecules on EVs enable targeted delivery of therapeutics into subcellular structures including mitochondria and nucleus, while minimizing off-target effects (35). Exosomal delivery of biologic materials can modulate disease processes by altering genetic profiles and biological responses of recipient cells (36).

Several studies have investigated the therapeutic potential of EVs as a vector for drug delivery in various kidney diseases. Tang et al. (37) have successfully produced macrophage-derived dexamethasone containing MVs through co-incubation of macrophages with dexamethasone. Delivery of these MVs into inflamed kidneys showed significant suppression of renal inflammation and fibrosis in lipopolysaccharide- or Adriamycin-induced murine model most likely through inhibition of NF- $\mathrm{KB}$ activity. In contrast with the traditional systemic glucocorticoid treatment which can cause hyperglycemia, infection, osteoporosis, and suppression of the hypothalamic-pituitary-adrenal (HPA) axis, chronic glucocorticoid treatment through these MVs showed no significant effect on serum glucose, HPA axis, bone metabolism, or immune response.

More recently, Yim et al. (38) have shown significant advancements in exosome research regarding its production efficiency and biological compatibility using "exosomes for protein loading via optically reversible protein-protein interactions" (EXPLOR), a novel optogenetically engineered exosome technology. By using this EXPLOR technology, Choi et al. (39) have successfully delivered super-repressor IKB-containing exosomes and shown their protective effects in a murine septic $\mathrm{AKI}$ model by inhibiting nuclear translocation of NF-KB. Recently, our group also reproduced the protective effect of exosomal super-repressor $1 \kappa B$ delivery treatment in an ischemic AKI model through its modulatory effect on inflammation and apoptosis (40).

As noncoding RNAs have gained more recognition for their important roles in various biological processes, delivery of noncoding RNAs as novel therapeutics for regulating renal disease progression using exosomes is in the limelight. Wang et al. (41) have shown that exosomal delivery of exogenous miRNAlet7c through engineered MSCs could alleviate renal fibrosis in a murine UUO model by suppressing TGF- $\beta$ signaling pathway. Their group also showed that exosomal delivery of miR-26a and miR-29 could attenuate renal fibrosis and muscle wasting in a murine UUO model $(42,43)$. These studies showed that exosome-carried miR-26a could limit renal fibrosis by directly suppressing connective tissue growth factor, while delivery of exosomal miR-29 could down-regulate pro-fibrotic proteins in TGF- $\beta$ pathway, attenuating the progression of renal fibrosis in UUO kidneys.

\section{CONCLUSIONS}

EVs carry high potentials as a novel therapeutic tool for modulation of disease courses and for drug delivery. However, 
application of EVs to renal diseases is still in its infancy stage despite the explosive advancement in EV research during the past decade. Clinical application of exosomes as a therapeutic tool has been mainly focused on cancer therapy and related studies in the nephrology field are relatively scarce. There are also several technical challenges to be surmounted including retaining high yields of pure exosomes, enhancing the capability of loading various cargoes, and improving targeting specificity (35). Therefore, further advancements of therapeutic application of EVs in various renal diseases need a multidisciplinary approach harnessed with better understanding of renal pathophysiology, multi-omics studies to find a novel therapeutic target, and supplementation of bioengineering technique to enhance the quality of exosomes as biocarriers. Further optimization of EV isolation techniques and scrupulous manipulation of genetic or protein compositions of EVs are mandatory to expand the therapeutic applicability of EVs. Rigorous in vivo and in vitro studies to better characterize the exact biological role of each EV treatment and evaluate potential off-target effects from EV treatment are required for individualized therapeutic application. This achievement needs to be followed by further validation through clinical trials and large-scale cohort studies before entering clinical application. These efforts will further extend the clinical applicability of exosomes as novel therapeutics of various renal diseases.

\section{ACKNOWLEDGEMENTS}

The authors thank Medical Illustration \& Design, part of the Medical Research Support Services of Yonsei University College of Medicine, for all artistic support related to this work.

\section{CONFLICTS OF INTEREST}

Tae Hyun Yoo is a Scientific Advisory Board member at ILIAS Biologics Inc. The authors have no additional financial interests.

\section{REFERENCES}

1. Karpman D, Ståhl AL and Arvidsson I (2017) Extracellular vesicles in renal disease. Nat Rev Nephrol 13, 545-562

2. Lipschutz JH (2019) The role of the exocyst in renal ciliogenesis, cystogenesis, tubulogenesis, and development. Kidney Res Clin Pract 38, 260-266

3. Lee SA, Choi C and Yoo TH (2021) Extracellular vesicles in kidneys and their clinical potential in renal diseases. Kidney Res Clin Pract 40, 194-207

4. Linxweiler J and Junker K (2020) Extracellular vesicles in urological malignancies: an update. Nat Rev Urol 17, 11-27

5. Jing H, Tang S, Lin S, Liao M, Chen H and Zhou J (2019) The role of extracellular vesicles in renal fibrosis. Cell Death Dis 10, 367

6. Behrens F, Holle J, Kuebler WM and Simmons S (2020) Extracellular vesicles as regulators of kidney function and disease. Intensive Care Med Exp 8, 22

7. Xu M, Yang Q, Sun X and Wang Y (2020) Recent advan- cements in the loading and modification of therapeutic exosomes. Front Bioeng Biotechnol 8, 586130

8. Bochon B, Kozubska M, Surygała G et al (2019) Mesenchymal stem cells-potential applications in kidney diseases. Int J Mol Sci 20, 2462

9. Chen W, Yan Y, Song C, Ding Y and Du T (2017) Microvesicles derived from human Wharton's Jelly mesenchymal stem cells ameliorate ischemia-reperfusion-induced renal fibrosis by releasing from $\mathrm{G} 2 / \mathrm{M}$ cell cycle arrest. Biochem J 474, 4207-4218

10. Zhang G, Zou X, Miao S et al (2014) The anti-oxidative role of micro-vesicles derived from human Wharton-Jelly mesenchymal stromal cells through NOX2/gp91(phox) suppression in alleviating renal ischemia-reperfusion injury in rats. PLoS One 9, e92129

11. Li X, Liao J, Su X et al (2020) Human urine-derived stem cells protect against renal ischemia/reperfusion injury in a rat model via exosomal miR-146a-5p which targets IRAK1. Theranostics 10, 9561-9578

12. Zhu G, Pei L, Lin F et al (2019) Exosomes from humanbone-marrow-derived mesenchymal stem cells protect against renal ischemia/reperfusion injury via transferring miR-199a3p. J Cell Physiol 234, 23736-23749

13. Pan T, Jia $P, C h e n ~ N$ et al (2019) Delayed remote ischemic preconditioning confers renoprotection against septic acute kidney injury via exosomal miR-21. Theranostics 9, 405-423

14. Grange C, Papadimitriou E, Dimuccio V et al (2020) Urinary extracellular vesicles carrying Klotho improve the recovery of renal function in an acute tubular injury model. Mol Ther 28, 490-502

15. Christov M, Neyra JA, Gupta S and Leaf DE (2019) Fibroblast growth factor 23 and Klotho in AKI. Semin Nephrol $39,57-75$

16. Dominguez JM 2nd, Dominguez JH, Xie D and Kelly KJ (2018) Human extracellular microvesicles from renal tubules reverse kidney ischemia-reperfusion injury in rats. PLoS One 13, e0202550

17. Brigstock DR (2021) Extracellular vesicles in organ fibrosis: mechanisms, therapies, and diagnostics. Cells 10, 1596

18. Ebrahim N, Ahmed IA, Hussien NI et al (2018) Mesenchymal stem cell-derived exosomes ameliorated diabetic nephropathy by autophagy induction through the mTOR signaling pathway. Cells 7, 226

19. Nagaishi K, Mizue Y, Chikenji T et al (2016) Mesenchymal stem cell therapy ameliorates diabetic nephropathy via the paracrine effect of renal trophic factors including exosomes. Sci Rep 6, 34842

20. Lindoso RS, Lopes JA, Binato R et al (2020) Adipose mesenchymal cells-derived EVs alleviate DOCA-salt-induced hypertension by promoting cardio-renal protection. Mol Ther Methods Clin Dev 16, 63-77

21. Cambier L, Giani JF, Liu W et al (2018) Angiotensin II-induced end-organ damage in mice is attenuated by human exosomes and by an exosomal Y RNA fragment. Hypertension 72, 370-380

22. Cantaluppi V, Medica D, Mannari C et al (2015) Endothelial progenitor cell-derived extracellular vesicles protect from complement-mediated mesangial injury in experimental anti-Thy1.1 glomerulonephritis. Nephrol Dial Transplant 
30, 410-422

23. Eirin A, Zhu XY, Puranik AS et al (2017) Mesenchymal stem cell-derived extracellular vesicles attenuate kidney inflammation. Kidney Int 92, 114-124

24. Song T, Eirin A, Zhu X et al (2020) Mesenchymal stem cell-derived extracellular vesicles induce regulatory $t$ cells to ameliorate chronic kidney injury. Hypertension 75, 12231232

25. Quaglia M, Dellepiane S, Guglielmetti G, Merlotti G, Castellano G and Cantaluppi V (2020) Extracellular vesicles as mediators of cellular crosstalk between immune system and kidney graft. Front Immunol 11, 74

26. Yu X, Huang C, Song B et al (2013) CD4 + CD25 + regulatory $\mathrm{T}$ cells-derived exosomes prolonged kidney allograft survival in a rat model. Cell Immunol 285, 62-68

27. Pang XL, Wang ZG, Liu L et al (2019) Immature dendritic cells derived exosomes promotes immune tolerance by regulating $\mathrm{T}$ cell differentiation in renal transplantation. Aging (Albany NY) 11, 8911-8924

28. Wu X, Yan T, Wang Z et al (2018) Micro-vesicles derived from human Wharton's Jelly mesenchymal stromal cells mitigate renal ischemia-reperfusion injury in rats after cardiac death renal transplantation. J Cell Biochem 119, 1879-1888

29. Nomura S, Takahashi N, Inami N et al (2004) Probucol and ticlopidine: effect on platelet and monocyte activation markers in hyperlipidemic patients with and without type 2 diabetes. Atherosclerosis 174, 329-335

30. Nomura S, Inami N, Shouzu A et al (2009) The effects of pitavastatin, eicosapentaenoic acid and combined therapy on platelet-derived microparticles and adiponectin in hyperlipidemic, diabetic patients. Platelets 20, 16-22

31. Dasgupta SK, Le A, Chavakis T, Rumbaut RE and Thiagarajan P (2012) Developmental endothelial locus-1 (Del-1) mediates clearance of platelet microparticles by the endothelium. Circulation 125, 1664-1672

32. Mossberg M, Ståhl AL, Kahn R et al (2017) C1-inhibitor decreases the release of vasculitis-like chemotactic endothelial microvesicles. J Am Soc Nephrol 28, 2472-2481

33. Liu X, Miao J, Wang C et al (2020) Tubule-derived exosomes play a central role in fibroblast activation and kidney fibrosis. Kidney Int 97, 1181-1195

34. Borges FT, Reis LA and Schor N (2013) Extracellular vesicles: structure, function, and potential clinical uses in renal diseases. Braz J Med Biol Res 46, 824-830

35. Jiang XC and Gao JQ (2017) Exosomes as novel bio-carriers for gene and drug delivery. Int J Pharm 521, 167-175

36. Kato M (2018) Noncoding RNAs as therapeutic targets in early stage diabetic kidney disease. Kidney Res Clin Pract 37, 197-209

37. Tang TT, Lv LL, Wang B et al (2019) Employing macrophage-derived microvesicle for kidney-targeted delivery of dexamethasone: an efficient therapeutic strategy against renal inflammation and fibrosis. Theranostics 9, 4740-4755

38. Yim N, Ryu SW, Choi $\mathrm{K}$ et al (2016) Exosome engineering for efficient intracellular delivery of soluble proteins using optically reversible protein-protein interaction module. Nat Commun 7, 12277

39. Choi H, Kim Y, Mirzaaghasi A et al (2020) Exosome-based delivery of super-repressor $\mathrm{I} \mathrm{K} \mathrm{B} \alpha$ relieves sepsis-associated organ damage and mortality. Sci Adv 6, eaaz6980

40. Kim S, Lee SA, Yoon $\mathrm{H}$ et al (2021) Exosome-based delivery of super-repressor $\mathrm{I} \mathrm{KB} \alpha$ ameliorates kidney ischemiareperfusion injury. Kidney Int 100, 570-584

41. Wang B, Yao K, Huuskes BM et al (2016) Mesenchymal stem cells deliver exogenous microRNA-let7c via exosomes to attenuate renal fibrosis. Mol Ther 24, 1290-1301

42. Zhang A, Wang $H$, Wang B, Yuan $Y$, Klein JD and Wang $\mathrm{XH}$ (2019) Exogenous miR-26a suppresses muscle wasting and renal fibrosis in obstructive kidney disease. FASEB J 33, 13590-13601

43. Wang H, Wang B, Zhang A et al (2019) Exosome-mediated miR-29 transfer reduces muscle atrophy and kidney fibrosis in mice. Mol Ther 27, 571-583

44. Gu D, Zou X, Ju G, Zhang G, Bao E and Zhu Y (2016) Mesenchymal stromal cells derived extracellular vesicles ameliorate acute renal ischemia reperfusion injury by inhibition of mitochondrial fission through miR-30. Stem Cells Int 2016, 2093940

45. Ju GQ, Cheng J, Zhong L et al (2015) Microvesicles derived from human umbilical cord mesenchymal stem cells facilitate tubular epithelial cell dedifferentiation and growth via hepatocyte growth factor induction. PLoS One 10, e0121534

46. Du T, Zhou J, Chen WX et al (2020) Microvesicles derived from human umbilical cord mesenchymal stem cells ameliorate renal ischemia-reperfusion injury via delivery of miR-21. Cell Cycle 19, 1285-1297

47. Collino F, Lopes JA, Corrêa S et al (2019) Adipose-derived mesenchymal stromal cells under hypoxia: changes in extracellular vesicles secretion and improvement of renal recovery after ischemic injury. Cell Physiol Biochem $52,1463-1483$

48. Xiang E, Han B, Zhang Q et al (2020) Human umbilical cord-derived mesenchymal stem cells prevent the progression of early diabetic nephropathy through inhibiting inflammation and fibrosis. Stem Cell Res Ther 11, 336

49. Grange C, Tritta S, Tapparo M et al (2019) Stem cellderived extracellular vesicles inhibit and revert fibrosis progression in a mouse model of diabetic nephropathy. Sci Rep 9, 4468

50. Nassar W, El-Ansary M, Sabry D et al (2016) Umbilical cord mesenchymal stem cells derived extracellular vesicles can safely ameliorate the progression of chronic kidney diseases. Biomater Res 20, 21

51. Jin J, Qian F, Zheng D, He W, Gong J and He Q (2021) Mesenchymal stem cells attenuate renal fibrosis via exosomes-mediated delivery of microRNA Let-7i-5p antagomir. Int J Nanomedicine 16, 3565-3578

52. Du T, Ju G, Zhou J et al (2021) Microvesicles derived from human umbilical cord mesenchyme promote M2 macrophage polarization and ameliorate renal fibrosis following partial nephrectomy via hepatocyte growth factor. Hum Cell 34, 1103-1113

53. Chen L, Wang Y, Li S et al (2020) Exosomes derived from GDNF-modified human adipose mesenchymal stem cells ameliorate peritubular capillary loss in tubulointerstitial fibrosis by activating the SIRT1/eNOS signaling pathway. Theranostics 10, 9425-9442 